\title{
NUMERICAL INVESTIGATION OF THE INTERACTIONS BETWEEN A LOW-HYPERSONIC SHOCK WAVE AND A WATER DROPLET: VOF AND DI METHODS COMPARISON
}

\author{
GWENC'HLAN TYMEN ${ }^{1,2}$, DAVID HEBERT ${ }^{1}$, JEAN-LUC RULLIER $^{1}$, \\ THIBAULT BRIDEL-BERTOMEU ${ }^{1}$, ISABELLE BERTRON ${ }^{1}$, SIMON PELUCHON ${ }^{1}$, \\ EMILIEN LESCOUTE ${ }^{3} \&$ FLORENT VIROT ${ }^{2}$ \\ ${ }^{1}$ CEA-CESTA, DAM, France. \\ ${ }^{2}$ ISAE-ENSMA, Institut Pprime, France. \\ ${ }^{3}$ CEA-DIF, DAM, France.
}

\begin{abstract}
In this paper, we present the hydrodynamic mechanisms which occur between a low-hypersonic shock wave and a millimetric water droplet. To do so, two numerical models, based respectively on the Volume of Fluid (VOF) and Diffuse Interfaces $(\boldsymbol{D I})$ approaches, are developed. The goal is to compare the results obtained with the models in order to evaluate which is the most accurate to describe the evolution of the physical phenomena. The studied Mach number and initial droplet diameter are 4.25 and $1.135 \mathrm{~mm}$, respectively. Each model allows the compressible Euler equations to be solved in a 2D-axisymmetric configuration. The evolution of both air and liquid phases is modelled by a stiffened gas equation of state. For qualitative validation, the numerical results are compared to experimental data recently presented in the literature. In this work, the authors used a shock tube test facility and a shadowgraph visualization technique to observe the phenomenology over a long time. Their investigation shows that the droplet deformation, detached bow shock and recompression waves are well captured by the two models until a Rayleigh dimensionless time of 1.5. Beyond this critical time, and up to 3 , some differences appear between the two numerical approaches, especially on the droplet deformation. Globally, the droplet deformation is better described with the VOF model, while the $\boldsymbol{D I}$ model appears to be more accurate when it comes to the evaluation of the position of the bow shock. In the discussion section, some ideas are proposed to improve the models.
\end{abstract}

Keywords: Diffuse Interfaces, shock wave, stiffened gas equations, Volume of Fluid, water droplet.

\section{INTRODUCTION}

Spray applications have been largely studied in the last decades because of their great importance in several industrial sectors such as aeronautics (rain erosion damage), nuclear or automotive (combustion process) [1-3]. Some authors like Kuhnke [4] and Mundo-Sommerfeld [5] proposed models, which are commonly used in the literature, to describe the interactions occurring between droplet and a solid wall.

Another topic is about the droplet(s) interactions, and more particularly water ones, with shock wave. In relation with this subject, Table 1 gives some literature references where the authors study experimentally the mechanisms appearing when a shock wave comes in contact with a droplet of water. Due to the important financial costs of such testing facilities and the recent great improvement of computing tools, numerical studies have become more and more present at the expense of experimental ones. The post-processing of simulations allows several physical variables to be studied in a way that experiences do not, due to the limitations of the sensors acquiring time, sensors position, visualization techniques and so on. Some works dealing with the interactions between a shock wave and a single water droplet are shown in Table 2.

To our knowledge, it seems that the majority of literature works (except the first experimental ones) do not deal with configurations where $\boldsymbol{M}$ is higher than 4. Especially, coupled 
Table 1: Some references of experimental works on shock wave and water droplet interactions.

\begin{tabular}{|c|c|c|c|c|}
\hline Author & Year & Reference & Droplet diameter (mm) & Mach (-) \\
\hline Engel & 1958 & [6] & $1.4-2.7$ & $1.3-1.7$ \\
\hline $\begin{array}{l}\text { Reinecke and } \\
\text { McKay }\end{array}$ & 1969 & [7] & $0.5-2.5$ & $3-12$ \\
\hline $\begin{array}{l}\text { Ranger and } \\
\text { Nicholls }\end{array}$ & 1970 & {$[8]$} & 0.75 & $2-5.7$ \\
\hline Boiko et al. & 1987 & [9] & $0.7-3.2$ & $2.37-3.8$ \\
\hline $\begin{array}{l}\text { Wierzba and } \\
\text { Takayama }\end{array}$ & 1988 & {$[10]$} & $1.03-4.3$ & $1.3-1.5$ \\
\hline $\begin{array}{l}\text { Yoshida and } \\
\text { Takayama }\end{array}$ & 1990 & {$[11]$} & 5.14 & 1.56 \\
\hline Chou et al. & 1997 & {$[12]$} & $0.59-1$ & $1.01-1.15$ \\
\hline Joseph et al. & 1999 & [13] & $2.5-2.6$ & $2-3$ \\
\hline Theofanous et al. & 2003 & [14] & 2.6 & 2 \\
\hline
\end{tabular}

Table 2: Some references of numerical works on shock wave and water droplet interactions.

\begin{tabular}{lllll}
\hline Author & Year & Reference & Droplet diameter $(\mathbf{m m})$ & Mach $(-)$ \\
\hline $\begin{array}{l}\text { Igra and } \\
\text { Takayama }\end{array}$ & 2001 & {$[15]$} & 6.4 & 1.47 \\
Nourgaliev et al. & 2004 & {$[16]$} & 6.4 & 1.47 \\
Chen & 2008 & {$[17]$} & $4.8-6.4$ & $1.3-1.47$ \\
Sanada et al. & 2011 & {$[18]$} & 4.8 & 1.47 \\
Meng and & 2015 & {$[2]$} & $4.8-6.4$ & $1.18-2.50$ \\
Colonius & & & & $1.75-2.4$ \\
Sembian et al. & 2016 & {$[19]$} & 22 & $1.47-3$ \\
Wang et al. & 2018 & {$[20]$} & 6.4 & \\
\hline
\end{tabular}

numerical/experimental studies for low-hypersonic cases $(\boldsymbol{M}=4-7)$ seem to be inexistent in literature, except a recent work [21] wherein the authors validate a Volume of Fluid (VOF) model by comparing the numerical results with experimental data for a $\boldsymbol{M}-4.25$ situation.

In this paper, two numerical models, based on a Diffuse Interfaces $(\boldsymbol{D I})$ and a $\boldsymbol{V O F}$ method, respectively, are used to simulate the interactions between a water droplet and a supersonic planar shock wave in a 2D-axisymmetric configuration. More particularly, the transient evolution of the bow shocks, recompression waves and droplet position/deformation is studied until $\boldsymbol{T}=3$. To validate the models, a comparison with experimental or numerical results from the literature is necessary. Concerning cases where $\boldsymbol{M} \leq 4$, data are already substantial in the literature. For this reason, we chose in this paper the reference case recently published in [21], where a low-hypersonic shock wave $(\boldsymbol{M}=4.25)$ hits a water droplet $\left(\varnothing_{i}=1.135 \mathrm{~mm}\right)$. 
The purpose of the present article is then to make a comparison between two numerical approaches ( $\boldsymbol{V O F}$ and $\boldsymbol{D I})$, in order to determine which is most appropriate to retranscribe, interpret and understand the experimental data presented in [21]. The numerical methods of both models are presented in section 2. Results obtained with the codes are then given in section 3 and discussed in section 4 by comparing them with experimental data from [21]. Conclusion and some perspectives on this work are finally proposed.

\section{NUMERICAL METHODS}

The first strategy to simulate the experimental work of [21] is to use a $\boldsymbol{D I}$ approach. In the literature, this method has been largely used to deal with multiphase flow configurations, and it appears that it can offer some advantages, such as its inherent conservative nature and the fact that the interfaces are simply obtained by numerical diffusion, and thus, a reconstruction algorithm is not necessary as in $\boldsymbol{V O F}$ methods. That is why, a simulation code based on this approach has been developed at Pprime laboratory to study, among other situations, shockdroplet interactions. The second strategy is to use a $\boldsymbol{V O F}$ approach, thanks to a code developed

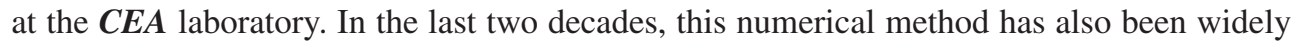
used by many authors to study several complex multiphase problems, such as boiling phenomenon [22] or bubble rising [23], for example, and gave good results. The code which is used here is based on a two-step 'Lagrangian + projection' principle, comparable to the one presented in [24]: a first Lagrangian calculation, giving the variables on a deformable mesh, is necessary at each time step, after which a projection process is realized to advect the variables on a fixed cartesian mesh. Generally speaking, the Lagrangian methods are well suited for multiphase flows having complex interfaces and are based on a deformable mesh. Besides, the Eulerian methods are well suitable for turbulent flows and use a fixed mesh [25]. The Lagrangian + projection strategy is thus used to avoid potential problems due to mesh degeneration caused by the Lagrangian method by using the projection process on a fixed mesh, while the interface-capturing property of the Lagrangian method is still conserved.

\subsection{The governing equations}

The Weber (ratio between inertial and capillary forces), Ohnesorge (ratio between viscous and capillary forces) and Reynolds (ratio between inertial and viscous forces) numbers are usually evaluated in compressible fluid mechanics. In the present situation, they respectively have an order of magnitude of $10^{5}, 10^{-3}$ and $10^{5}$. Therefore, with regard to these values, the assumption is made to simplify the models by not taking into account the surface tension and viscosity terms [17]. In the $\boldsymbol{D I}$ model, the set of equations which are used is similar to the one presented in [26]. Concerning the $\boldsymbol{V O F}$ model, the formulation describing the compressible Euler equations has also been previously studied in the literature [27]. In the two models, the evolutions of both liquid and gas phases are modelled by the Stiffened-Gas E.O.S, which enables the systems to be closed, by defining the pressure term. The E.O.S parameters used are the ones of [16] for a shock tube configuration and set constant during the simulations.

\subsection{Numerical discretization}

For the $\boldsymbol{D I}$ model, a second-order RK-TVD scheme is used to temporally discretize the equations [28]. The spatial discretization is ensured by a MUSCL scheme coupled to a HLLC Riemann solver, which is a good choice to capture the interfaces based on literature data [20]. 
Note that a minmod limiter is used to avoid the occurrence of non-physical oscillations. In the VOF model, our MYR numerical scheme, variant of the BBC one [27], is used. With this method, the discretization is done differently depending on the variables at the vertices of the cells for the velocity components (and not at the edges as in the BBC scheme) and at the centre of the cells for the other variables (volume, density, pressure, energy) [29, 30]. As aforementioned, a Lagrangian process is necessary to evaluate the variables before the projection. During this step, the MYR scheme is coupled to a temporal discretization realized with the Young's method which is based on a predictor-corrector strategy. Besides, the spatial discretization is performed with the Wilkins' method [31].

\subsection{Interface capture}

In $\boldsymbol{D I}$ methods, the position of the interface between two fluids is not precisely tracked, contrary to the $\boldsymbol{V O F}$ approach. In fact, it is obtained thanks to the numerical diffusion which creates an artificial non-physical mixture zone between different species and/or phases [32]. With a $\boldsymbol{V O F}$ method, a reconstruction process is needed in each cell of the computational domain to obtain a sharp interface between the phases before advecting it [33]. Here, it is reconstructed with the Mosso's method [34]. In addition, to handle shocks and provide some stability to the numerical scheme, a pseudo-viscosity term is added to the pressure one [27].

\subsection{Initial conditions}

Since $\boldsymbol{M}$ is known in the studied case, as well as the variables at rest, the Rankine-Hugoniot relations [35] have been used to determine the value of the shocked state variables, in order to initialize the model. Here, the values of $P_{0}$ and $\rho_{0}$ have been tuned in order to obtain a sound velocity at rest $c_{0}$ of $340 \mathrm{~m} \mathrm{~s}^{-1}$ (commonly used in the literature [36]). They are then $101325 \mathrm{~Pa}$ and $1.227 \mathrm{~kg} \mathrm{~m}^{-3}$ air, respectively. The pressure of the liquid phase is also set to $101325 \mathrm{~Pa}$, whereas its density is $1000 \mathrm{~kg} \mathrm{~m}^{-3}$.

\subsection{Computational domain and meshing}

For the case considered here, using a 3D mesh was out of question to reduce the computational costs. That is why, a structured and regular 2D-cartesian mesh is chosen to model the configuration. As seen in Fig. 1, only half of the drop is modelled and an axial symmetry is applied to reduce the computational costs. In order to observe the evolution of the phenomena at both long term and long distance, the size of the domain has been set to $30 \times 20 \mathrm{~mm}$ (with the reflection symmetry). The mesh consists here of 2400 cells along the $(\mathrm{O}, \mathrm{x})$ direction and 800 cells along the $(\mathrm{O}, \mathrm{y})$ direction, which represents a resolution of $12.5 \mu \mathrm{m}$ cell $^{-1}$ and 90 cells in the droplet diameter.

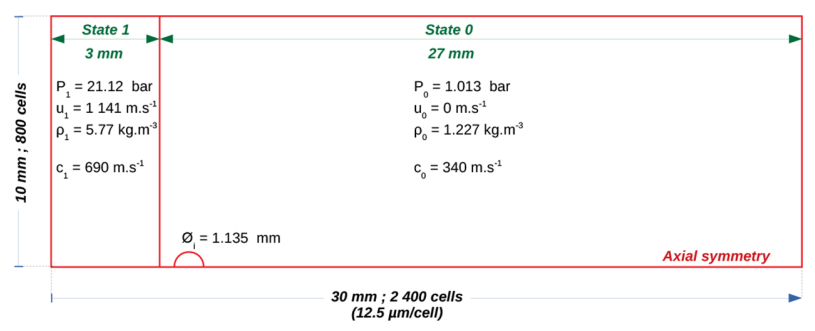

Figure 1: Geometrical domain and initial conditions. 


\section{RESULTS AND QUALITATIVE COMPARISON}

In this section, the results obtained with our numerical tools (VOF and $\boldsymbol{D I}$ methods) are compared to the experimental data from [21]. The most relevant observations are listed in Tables $3-5$, before being discussed in section 5 . As the physical phenomena have been already presented in [21], the goal is here to study only the qualitative accuracy of the two numerical approaches. To ease the comparison, the transient study is divided here into three main ranges:

- early stage: $T \leq 1$,

- medium stage: $1 \leq T \leq 2$ and

- late stage: $2 \leq T$.

$T$ represents the dimensionless Rayleigh time, based on the physical time $t$, the matter velocity $u_{1}$, the initial droplet diameter $\varnothing_{i}$ and the phases density $\rho$ [37]. In the following, the experimental data are shown in white/grey shades, on top of which the numerical results are overlaid. The $\boldsymbol{V O F}$ and $\boldsymbol{D I}$ are represented on the bottom and the top sides, respectively. The gradients of the velocity field are coloured in orange (upper part), while the gradients of the density are in blue (lower part). Every numerical result has been synchronized with the experiment at the precise studied time.

\subsection{Early stage: $T \leq 1$}

In this section, the interactions occurring when the shock wave impacts the droplet are studied until $T \leq 1$. The qualitative comparison between the numerical models and the experimental data is shown in Fig. 2 and yields some interesting results which are given in Table 3.

According to the qualitative analysis which has been made to study the evolutions of bow shock, recompression wave and droplet deformation until $T=0.90$, two main conclusions should be pointed out: the code-to-code comparison shows no significant differences between the $\boldsymbol{V O F}$ and $\boldsymbol{D I}$ approaches; the experimental data are well described by the two numerical methods that are therefore validated.

\subsection{Medium stage: $1 \leq T \leq 2$}

When $T>1$, some differences begin to appear between the two numerical approaches, as observed in the qualitative visualizations on Fig. 3. Again, several points stand out and are presented in Table 4.

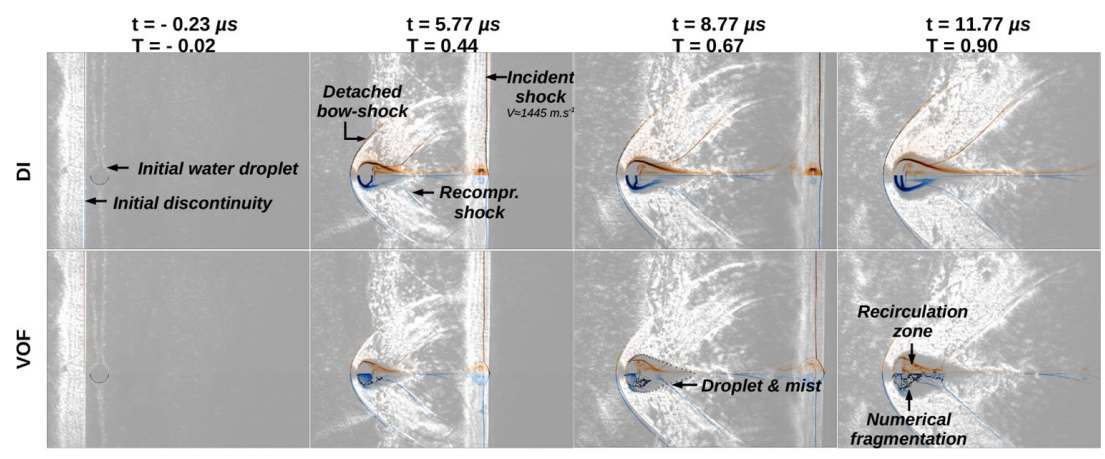

Figure 2: Overlay of numerical results with experimental data for $\mathrm{T}<1$.

Top: DI - Bottom: VOF; Upper and lower halves: velocity and density gradient 
Table 3: Numerical results compared to experimental data for $T<1$.

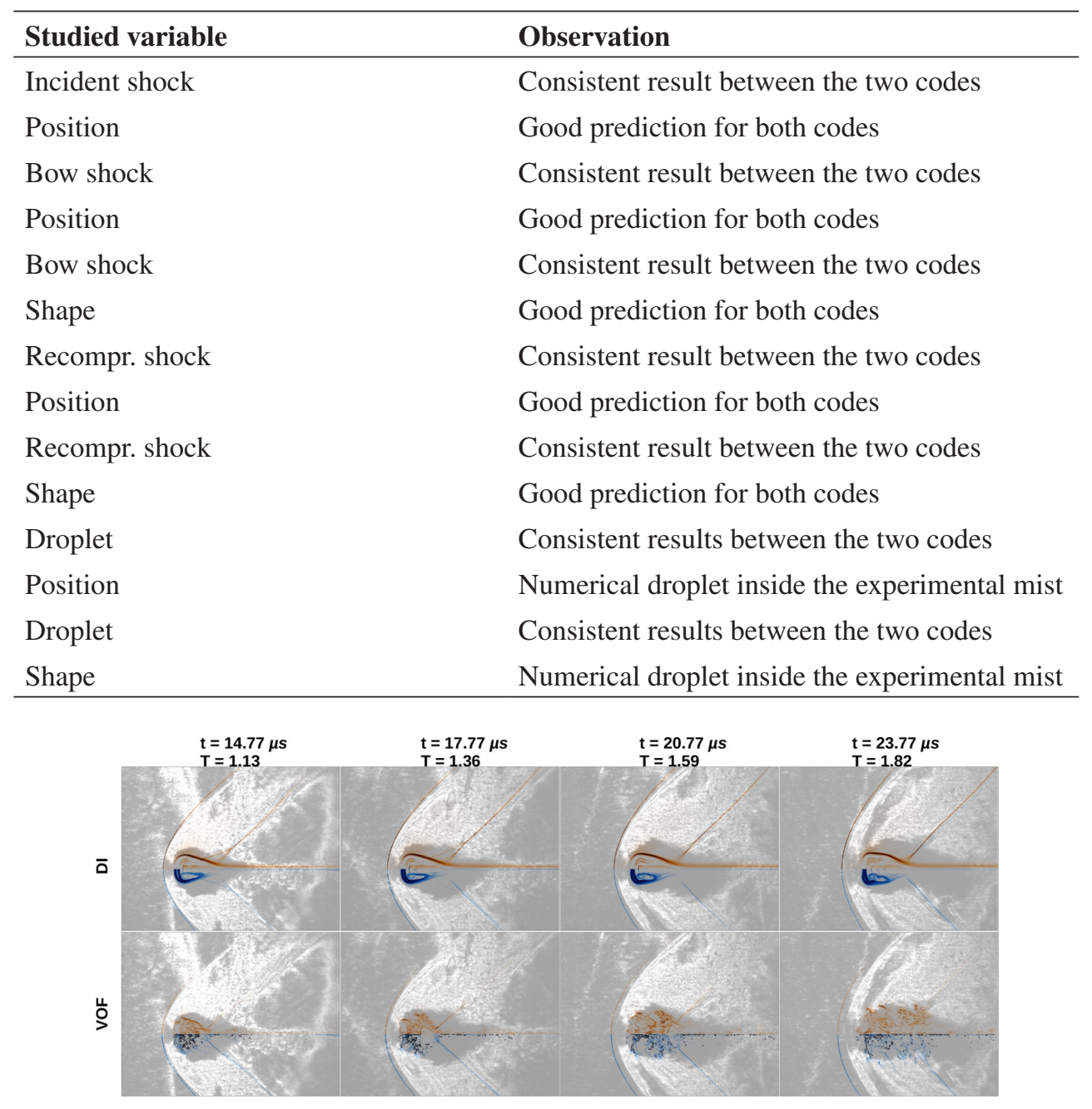

Figure 3: Overlay of numerical results with experimental data for $1<\mathrm{T}<2$.

Top: DI; bottom: VOF; upper and lower halves: velocity and density gradient.

According to these qualitative analyses for $1<T<2$, here are the main conclusions: the bow-shock position is consistent between the two codes, but the $\boldsymbol{D I}$ model seems to give better results from $T>1.13$; the droplet position is consistent between the two codes, but its shape differs. Therefore, the $\boldsymbol{V O F}$ results are closer to the experimental data than the $\boldsymbol{D I}$ ones; overall, there is a good agreement between the codes.

\subsection{Late stage: $2 \leq \mathrm{T}$}

Finally, a qualitative comparison of the models with experimental data when $T$ is greater than 2 is shown in Fig. 4. The main observations that emerge are listed in Table 5. 
Table 4: Numerical results compared to experimental data for $1<T<2$.

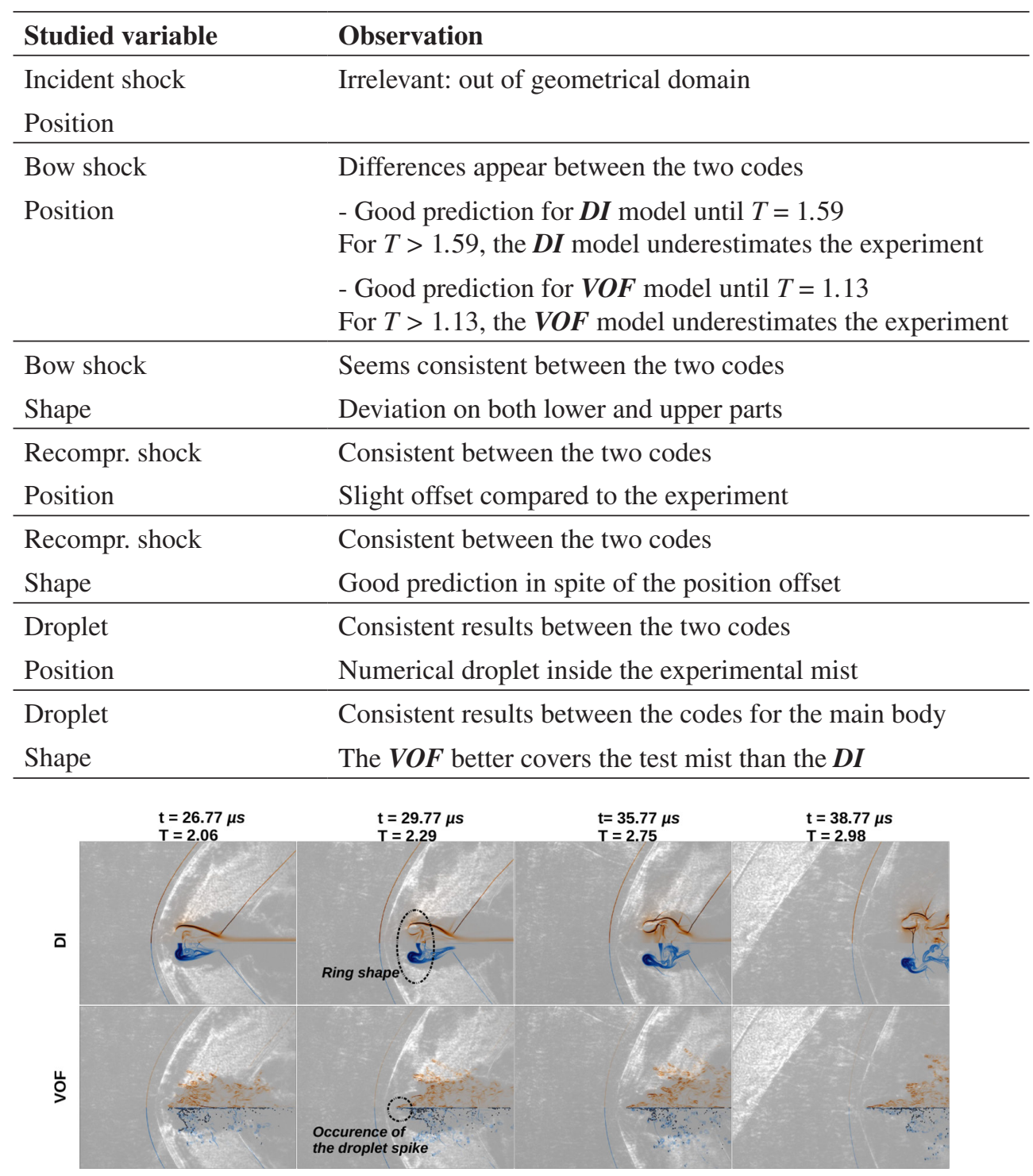

Figure 4: Comparison of numerical results with experimental data for $2>T$.

Top: DI - Bottom: VOF ; Upper and lower halves: velocity and density gradient

Based on the observations given in Table 5, it appears that the code-to-code comparison still shows a slight difference for the evaluation of the bow-shock position: the $\boldsymbol{D I}$ is closer to the experimental data than the $\boldsymbol{V O F}$, but anyways it seems that none of the models match with experimental data anymore; the droplet seems to be better evaluated by the $\boldsymbol{V O F}$ model, for the front position as well as its global shape; both codes do not reproduce the experimental bow-shock shape, which becomes sharp for late times. 
Table 5: Numerical results compared to experimental data for $T>2$.

\begin{tabular}{|c|c|}
\hline Studied variable & Observation \\
\hline Incident shock & Irrelevant: out of geometrical domain \\
\hline \multicolumn{2}{|l|}{ Position } \\
\hline Bow shock & Both codes underestimate the experiments \\
\hline Position & The deviation increases over time \\
\hline Bow shock & Consistent results between the two codes \\
\hline Shape & Large deviation compared to the test from $T=2.29$ \\
\hline Recompr. shock & Consistent results between the two codes until $T=2.29$ \\
\hline Position & $\begin{array}{l}\text { Both codes underestimate the experiments } \\
\text { For } T>2.29 \text {, it is not evaluable anymore }\end{array}$ \\
\hline Recompr. shock & Consistent results between the two codes \\
\hline Shape & Both codes underestimate the experiments \\
\hline Droplet & Differences appear between the two codes \\
\hline Position & $\begin{array}{l}\text { VOF predicts a spike consistent with experiments } \\
\boldsymbol{D I} \text { gives a faster droplet displacement }\end{array}$ \\
\hline Droplet & Large differences between the two codes: \\
\hline \multirow[t]{3}{*}{ Shape } & - with $\boldsymbol{D I}$, the droplet is composed of a ring and a film and \\
\hline & $\begin{array}{l}\text { - with } \boldsymbol{V O F} \text {, the droplet stretches and forms a spike along the } \\
(\mathrm{O}, \mathrm{x}) \text { axis. }\end{array}$ \\
\hline & The $\boldsymbol{V O F}$ stills better covers the test mist than the $\boldsymbol{D I}$ \\
\hline
\end{tabular}

\section{DISCUSSION}

In this section, the qualitative results presented in section 3 are discussed in order to give some explanation concerning the validity of the numerical models by referring to several literature works. The differences observed between the two models are explained, and some perspectives to improve the modelling are proposed.

\subsection{The incident shock displacement}

The experimental data are well described over time by the $\boldsymbol{V O F}$ and $\boldsymbol{D I}$ methods, meaning that the initial conditions $\left(P_{1}, u_{1}\right.$ and $\rho_{1}$ given in Fig. 1$)$ determined by the Hugoniot relations are coherent. Within the limit of the available experimental data (until $T=0.67$ ), both models give satisfactory results.

\subsection{The bow-shock position}

Until $T=1.13$, both $\boldsymbol{V O F}$ and $\boldsymbol{D I}$ models match the experimental data in a really good way. However, beyond this critical time, some differences are visible. It is interesting to note that they seem to occur when the experimental droplet breakup is initiated. Indeed, according to Pilch [38], this process starts at $T=1.25$ for high $\boldsymbol{W e}$. It is clear that a deviation from the 
experimental results occurs for both models and tends to grow over time. This deviation can potentially be explained by the fact that air is here modelled by a perfect gas law, where $\gamma$ remains constant at 1.4, and not a real gas one. Some literature works, as the ones of Vallerani in 1969 [39], give the evolution of the specific heat ratio over a large $\boldsymbol{M}$ range. According to their results, it appears that at $\boldsymbol{M}=4.26$ and the corresponding $\gamma$ is rather 1.38. To analyse the influence of this value, new calculations have been carried out for both models with $=1.38$. The benefit, not shown here, is obvious: the numerical bow-shock position is closer to the experimental one for the entire range $T=[0 ; 2.98]$.

\subsection{The bow-shock shape}

Globally, even if the bow-shock position obtained numerically is not entirely satisfactory, especially over a long time $T$, it seems that the shape of the bow shock is pretty well described by both codes until $T=2.29$. Beyond this time, the experimental bow shock is much sharper than the numerical ones. Again, this deviation could potentially be explained by the absence of a real gas law to model the fluids. Indeed, in the numerical work presented in [21], this kind of law (which considered a variable $\gamma$, vibration, ionization and dissociation) is used and the results are clearly better. This analysis shows one of the limitations of the use of a perfect gas law.

\subsection{The recompression shock}

On the whole, the recompression shock shape and position are similar for both codes and seem to be well described when comparing them to the test for all time steps until $T=2.52$. Beyond this time, the experiment does not clearly show the recompression shock anymore.

\subsection{The droplet position}

The front droplet position seems to be better evaluated by the $\boldsymbol{V} \boldsymbol{O} \boldsymbol{F}$ method when $T>2$, based on the comparison with the experimental data from [21]. This observation can be linked to those of [40], where Mirjalili showed that a $\boldsymbol{V O F}$ approach was more accurate than a $\boldsymbol{D I}$ approach to capture the interface position on an equivalent mesh, for several multiphase configurations. This author then showed that $\mathbf{V O F}$ and $\boldsymbol{D I}$ methods provide actually equivalent results when the $\boldsymbol{D I}$ mesh resolution is twice that of $\boldsymbol{V O F}$ [40]. It appears that both codes predict the bow shock to be at the same location, in spite of the differences in droplet shape. This observation is, however, to be considered with care as it is known, and many works such as [41] show, that the bow-shock position should be strongly dependent on the droplet shape.

\subsection{The droplet shape}

Even if the results are consistent between the $\boldsymbol{V O F}$ and $\boldsymbol{D I}$ methods for the early times, it seems difficult to judge their validity as the experimental droplet body is actually hidden in the mist. In the figures, it is still observable that for both models, the back of the droplet is deformed before the front. This is due to a weaker density in this region, because an expansion wave has been previously transmitted inside the media, as explained in [15]. Although there are only a few, the shapes obtained here can be compared to some literature papers: at $T=0.44$, even if the initial state is different, the droplet has a shape which clearly reminds the 
'muffin' shape of Meng and Colonius (Fig. 6 in [37] for $T=0.344$ ) or those obtained by Theofanous (Fig. 3 in [3] for $T=0.36$ ): the front of the droplet remains spherical, whereas the back is compressed into a flat plane. At this time, the experimental work of [21] shows a mist, which is, in fact, composed of liquid fragments, previously stripped off the water droplet. Then, the fragments generate a kind of curtain around a cavity as explained in [37]; the droplet is next deformed into a liquid sheet at $T=0.90$, which has been also obtained by Meng and Colonius (Fig. 6 in [37] for $T=0.935$; in this paper, this regime obtained numerically has been compared to an experimental mist and considered as validated). When $T>1.5$, it is clear that $\boldsymbol{V O F}$ and $\boldsymbol{D I}$ methods give different results concerning the shape of the droplet. In this configuration, where $\boldsymbol{M}$ and $\boldsymbol{W e}$ numbers are both high, the literature data is not abundant. Moreover, in the experimental work of [21], the droplet is hidden in the mist, which makes it impossible to know its shape. Having this in mind, our numerical results predicting the droplet shape are only compared with this mist. Nevertheless, knowing that the experimental droplet is not perfectly symmetric, the fact that the $2 \mathrm{D}$-axisymmetric numerical droplet is always inside the experimental mist for both codes is encouraging.

\section{CONCLUSION AND PERSPECTIVES}

In this paper, the results of two numerical models, based on the $\boldsymbol{V O F}$ and $\boldsymbol{D I}$ approaches, respectively, have been investigated to observe the phenomena which appear when a low-hypersonic shock wave $(\boldsymbol{M}=4.25)$ impacts a millimetric water droplet $\left(\varnothing_{i}=1.135\right.$ $\mathrm{mm})$. The 2D-axisymmetric simulations which have been carried out are in good agreement with experimental data from the literature until a dimensionless time of 1.5. Beyond this value, some deviation appears between the models themselves and with the experiments. Overall, the $\boldsymbol{V O F}$ method is more accurate for the prediction of the droplet shape, whereas the $\boldsymbol{D I}$ method is better for the prediction of bow-shock position, on an equivalent mesh. Several options have been discussed and could improve the numerical results by modifying the behavioural law of air, for example. Indeed, $\gamma$ has been set to 1.4 in the presented work, but some studies in the literature show that this classical value is barely correct for such high $\boldsymbol{M}$-range. Thus, a more physical model for air could be used to deal with this configuration, rather than a perfect gas law. In addition, it must be recalled that one of the basic assumptions is also a source of errors: the viscosity and surface tension terms were not considered for the calculations because of the initial values of Weber, Ohnesorge and Reynolds numbers. Some authors, such as [37], conclude that these parameters' effects become important when the studied time is important. Their absence in our numerical model could then generate some unwelcome phenomena or prevent us from observing the right behaviour. However, modelling these additional terms would require the simulation of length scales smaller than the current cell size, as discussed in [21], and this would then greatly increase the costs: a balance has to be found. The numerical modelling itself can also be a source of discussions. Indeed, as presented in section 3, the MYR scheme has been used for the VOF model. Others, such as Godunov or BBC [27], could be tried to evaluate more accurately the influence of the scheme on the experimental deviation. Furthermore, the $\boldsymbol{D I}$ calculation has been performed with a minmod limiter, which is known as quite diffusive in the literature [42]. Van Leer or Superbee limiters [43], for example, could potentially improve this aspect. To improve the results of $\boldsymbol{D I}$, some anti-diffusion methods could also be studied and taken into account in the model [44]. The modelling of the secondary atomization would also be a source of improvement in the model to obtain more details during the fragmentation [45]. Using another numerical approach, such as the 
commonly used 'mixed Euler-Lagrange' approach for industrial applications [47], could also be a part of a future work. Indeed, it has been recently validated by Hrebtov when a shock wave passes through a cloud of droplets (Euler for the carrier air and Lagrange for the dispersed water phase), giving them encouraging perspectives [48]. That is why, some studies could be carried out to observe the relevance of this approach when applied to our configuration. Finally, some 3D simulations could be carried out to help the scientific community better understand the phenomena occurring when $\mathrm{T}$ becomes important.

\section{ACKNOWLEDGEMENTS}

This work was supported by the SEIGLE project of Re'gion Nouvelle-Acquitaine. For the DI model, the computations were performed using HPC resources from the cluster of Pprime, the Me'socentre de Calcul Poitevin.

\section{REFERENCES}

[1] Jenkins, D.C. \& Bowden, F.P., Practical aspects of rain erosion of aircraft and missiles. Philosophical Transactions of the Royal Society of London Series A, Mathematical and Physical Sciences, 260(1110), pp. 153-160, 1966. https://doi.org/10.1098/ rsta. 1966.0040

[2] Meng, J.C. \& Colonius, T., Numerical simulations of the early stages of high-speed droplet breakup. Shock Waves, 25(4), pp. 399-414, 2015. https://doi.org/10.1007/ s00193-014-0546-Z

[3] Theofanous, T.G. \& Li, G.J., On the physics of aerobreakup. Physics of Fluids, 20(5), p. 052103, 2008. https://doi.org/10.1063/1.2907989

[4] Kuhnke, D., Spray wall interaction modeling by dimensionless data analysis. PhD Thesis, 2004.

[5] Mundo, C., Droplet-wall collisions: Experimental studies of the deformation and breakup process. International Journal of Multiphase Flow, 21(2), pp. 151-173, 1995. https://doi.org/10.1016/0301-9322(94)00069-v

[6] Engel, O., Fragmentation of waterdrops in the zone behind an air shock. Journal of Research of the National Bureau of Standards, 60(3), p. 245, 1958. https://doi. org/10.6028/jres.060.029

[7] Reinecke, W.G. \& McKay, W.L., Experiments on water drop breakup behind mach 3 to 12 shocks. Report AVATD-0172-69-RR, 1969.

[8] Ranger \& Nicholls, Water droplet breakup in high speed airstreams. 3rd Inter Conf Rain Erosion and Allied Phenomena, 1970.

[9] Boiko, V.M., Papyrin, A.N. \& Poplavskii, S.V., Dynamics of droplet breakup in shock waves. Journal of Applied Mechanics and Technical Physics, 28(2), pp. 263-269, 1987. https://doi.org/10.1007/bf00918731

[10] Wierzba, A. \& Takayama, K., Experimental investigation of the aerodynamic breakup of liquid drops. AIAA Journal, 26(11), pp. 1329-1335, 1988. https://doi. org/10.2514/3.10044

[11] Yoshida, T. \& Takayama, K., Interaction of liquid droplets with planar shock waves. Journal of Fluids Engineering, 112(4), pp. 481-486, 1990. https://doi. org/10.1115/1.2909431

[12] Chou, W.H., Hsiang, L.P. \& Faeth, G., Temporal properties of drop breakup in the shear breakup regime. International Journal of Multiphase Flow, 23(4), pp. 651-669, 1997. https://doi.org/10.1016/s0301-9322(97)00006-2 
[13] Joseph, D., Belanger, J. \& Beavers, G., Breakup of a liquid drop suddenly exposed to a high-speed airstream. Int Journal of Multiphase Flow, 25(6), pp. 1263-1303, 1999. https://doi.org/10.1016/s0301-9322(99)00043-9

[14] Theofanous, T., Li, G. \& Dinh, N., Rayleigh-taylor and kelvin-helmholz instabilities in aerobreakup. American Physical Society, 2003.

[15] Igra, D. \& Takayama, K., Experimental and numerical study of the initial stages in the interaction process between a planar shock wave and a water column. Proceedings of 23rd International Symposium on Shock Waves, p. 8, 2001.

[16] Nourgaliev, R., Dinh, N. \& Theofanous, T., Direct numerical simulation of compressible multiphase flows. International Conference on Multiphase Flow, ICMF04, p. 18, 2004.

[17] Chen, H., Two-dimensional simulation of stripping breakup of a water droplet. AIAA Journal, 46, pp. 1135-1143, 2008. https://doi.org/10.2514/1.31286

[18] Sanada, T., Ando, K. \& Colonius, T., A computational study of high-speed droplet impact. Fluid Dynamics and Materials Processing, p. 12, 2011. https://doi.org/10.4028/ www.scientific.net/ssp.187.137

[19] Sembian, S., Liverts, M., Tillmark, N. \& Apazidis, N., Plane shock wave interaction with a cylindrical water column. Physics of Fluids, 28, p. 056102, 2016. https://doi. org/10.1063/1.4948274

[20] Wang, T., Liu, N., Yi, X., Lu, X. \& Wang, P., Numerical study on shock/droplet interaction before a standing wall. CiCP, 23(4), 2018. https://doi.org/10.4208/cicp.oa-20160254

[21] He'bert, D., Rullier, J.L., Chevalier, J.M., Bertron, I., Lescoute, E., Virot, F. \& El-Rabii, H., Investigation of mechanisms leading to water drop breakup at mach 4.4 and weber numbers above 105. SN Applied Sciences, 2(1), p. 69, 2019. https://doi.org/10.1007/ s42452-019-1843-z

[22] Tymen, G., Allanic, N., Sarda, A., Mousseau, P., Plot, C., Madec, Y. \& Caltagirone, J., Temperature mapping in a two-phase water-steam horizontal flow. Experimental Heat Transfer, 31(4), pp. 317-333, 2018. https://doi.org/10.1080/08916152.2017.1410505

[23] Cano-Lozano, The use of volume of fluid technique to analyze multiphase flows: Specific case of bubble rising in still liquids. Applied Mathematical Modelling, 2014.

[24] Bernard-Champmartin, A. \& Vuyst, F.D., A low diffusive lagrange-remap scheme for the simulation of violent air-water free-surface flows. Journal of Computational Physics, 274, pp. 19-49, 2014. https://doi.org/10.1016/j.jcp.2014.05.032

[25] Gasc, T., Improving numerical methods on recent multi-core processors. Application to Lagrange-Plus-Remap hydrodynamics solver. Thesis, 2016.

[26] Chinnayya, A., Daniel, E. \& Saurel, R., Modelling detonation waves in heterogeneous energetic materials. Journal of Computational Physics, 196(2), pp. 490-538, 2004. https://doi.org/10.1016/j.jcp.2003.11.015

[27] Champmartin, A., Mode'lisation et e'tude nume'rique d'e'coulements diphasiques. Thesis, 2011.

[28] Shu, C.W. \& Osher, S., Efficient implementation of essentially non-oscillatory shockcapturing schemes. Journal of Computational Physics, 77(2), pp. 439-471, 1988.

[29] Williams, R., Sub-grid properties and artificial viscous stresses in staggered-mesh schemes. Journal of Computational Physics, 374, pp. 413-443, 2018. https://doi. org/10.1016/j.ijimpeng.2010.07.003 
[30] Dawes, A., Parallel multi-dimensional and multi-material eulerian staggered mesh schemes using localised patched based adaptive mesh refinement (amr) for strong shock wave phenomena. Adaptative Mesh Refinement - Theory and Applications, 41, 2005.

[31] Wilkins, M.L., Use of artificial viscosity in multidimensional fluid dynamic calculations. Journal of Computational Physics, 36(3), pp. 281-303, 1980. https://doi. org/10.1016/0021-9991(80)90161-8

[32] Richard, S., Petitpas, F. \& Berry, R.A., Simple and efficient relaxation methods for interfaces separating compressible fluids, cavitating flows and shocks in multiphase mixtures. Journal of Computational Physics, 228(5), pp. 1678-1712, 2009. https://doi. org/10.1016/j.jcp.2008.11.002

[33] Mirjalili, S., Jain, S.S. \& Dodd, M.S., Interface-capturing methods for two-phase flows: An overview and recent developments. Center for Turbulence Research - Annual Research Brief, p. 19, 2017.

[34] Mosso, S. \& Clancy, S., Geometrically derived priority system for youngs' interface reconstruction. Tech report, Los Alamos National Laboratory, 1995.

[35] Leboucher, J.C., Re'alisation et performances d'un tube a` choc a` deux diaphragmes. Thesis, p. 111, 1973.

[36] Wang, E. \& Shukla, A., Analytical and experimental evaluation of energies during shock wave loading. International Journal of Impact Engineering, 37(12), pp. 11881196, 2010. https://doi.org/10.1016/j.ijimpeng.2010.07.003

[37] Meng, J.C. \& Colonius, T., Numerical simulation of the aerobreakup of a water droplet. Journal of Fluid Mechanics, 835, p. 1108-1135, 2018. https://doi.org/10.1017/ jfm.2017.804

[38] Pilch, M. \& Erdman, C., Use of breakup time data and velocity history data to predict the maximum size of stable fragments for acceleration-induced breakup of a liquid drop. International Journal of Multiphase Flow, 13(6), pp. 741-757, 1987. https://doi. org/10.1016/0301-9322(87)90063-2

[39] Vallerani, E., An "ideal equivalent gas method" for the study of shock waves in supersonic real gas flows. Meccanica, 4(3), pp. 234-249, 1969. https://doi.org/10.1007/ bf02133438

[40] Mirjalili, S., Ivey, C.B. \& Mani, A., Comparison between the diffuse interface and volume of fluid methods for simulating two-phase flows. International Journal of Multiphase Flow, 116, pp. 221-238, 2019. https://doi.org/10.1016/j.ijmultiphaseflow.2019.04.019

[41] Baals, D.D. \& Corliss, W.R., Wind tunnels of nasa. Technical Report-Research and support facilities, 1981.

[42] Sharma, P. \& Hammett, G.W., Preserving monotonicity in anisotropic diffusion. Journal of Computational Physics, 227(1), pp. 123-142, 2007. https://doi.org/10.1016/j. jcp.2007.07.026

[43] Pudasaini, S. \& Kröner, C., Shock waves in rapid flows of dense granular materials: Theoretical predictions and experimental results. Physical Review E, Statistical, Nonlinear, and Soft Matter Physics, 78, p. 041308, 2008. https://doi.org/10.1103/physreve.78.041308

[44] Kokh, S. \& Lagoutie're, F., An anti-diffusive numerical scheme for the simulation of interfaces between compressible fluids by means of a five-equation model. Journal of Computational Physics, 229, pp. 2773-2809, 2010. https://doi.org/10.1016/j. jcp.2009.12.003 
[45] Umemura, A. \& Shinjo, J., Detailed sgs atomization model and its implementation to two-phase flow les. Combustion and Flame, 195, pp. 232-252, 2018. https://doi. org/10.1016/j.combustflame.2018.01.026

[46] Vajda, B., Les`nik, L., Bombek, G., Bilus`, I., Z` unic`吕., S`kerget, L., Hoc`evar, M., S`irok, B. \& Kegl, B., The numerical simulation of biofuels spray. Fuel, 144, pp. 71-79, 2015. https://doi.org/10.1016/j.fuel.2014.11.090

[47] Pogorevc, P., Kegl, B. \& S`kerget, L., Diesel and biodiesel fuel spray simulations. Energy and Fuels - ENERG FUEL, 22, 2008.

[48] Hrebtov, M., Mixed lagrangian-eulerian simulation of interaction between a shockwave and a cloud of water droplets. Journal of Engineering Thermophysics, 29, 2020. 